PROCEEDINGS OF THE

AMERICAN MATHEMATICAL SOCIETY

Volume 127, Number 2, February 1999, Pages 509-517

S 0002-9939(99)04870-4

\title{
A FACTORIZATION THEOREM FOR THE DERIVATIVE OF A FUNCTION IN $H^{p}$
}

\author{
WILLIAM S. COHN
}

(Communicated by Theodore W. Gamelin)

\begin{abstract}
We show that a function $G$ is the derivative of a function $f$ in the Hardy space $H^{p}$ of the unit disk $D$ for $0<p<\infty$ if and only if $G=F \Phi^{\prime}$ where $F \in H^{p}$ and $\Phi \in B M O A$. Here, $F$ can be chosen to be non-vanishing, $\|\Phi\|_{B M O A} \leq 1$, and $\|F\|_{H^{p}} \leq C\|f\|_{H^{p}}$. As an application, we characterize positive measures $\mu$ on the unit disk such that the operator $L_{\mu} g(\zeta)=\int_{D} g(z) \frac{d \mu(z)}{(1-\zeta \bar{z})^{2}}$ is bounded from the tent space $T_{\infty}^{p}$ to $H^{p}$, where $\frac{1}{2}<p<\infty$.
\end{abstract}

Let $D$ be the unit disk in the complex plane and suppose that $T$ is its boundary, the unit circle. For $\zeta \in T$, let $\Gamma(\zeta)$ be the non-tangential approach region

$$
\Gamma(\zeta)=\left\{z \in D:|1-z \bar{\zeta}|<\left(1-|z|^{2}\right)\right\} .
$$

Associated with the approach regions $\Gamma(\zeta), \zeta \in T$, are the maximal functions $N u$ and $A u$. Recall that for a function $u$ defined on $D$

$$
N u(\zeta)=\sup _{z \in \Gamma(\zeta)}|u(z)|
$$

and

$$
A u(\zeta)=\left(\int_{\Gamma(\zeta)}|u(z)|^{2} \frac{d a(z)}{(1-|z|)^{2}}\right)^{\frac{1}{2}},
$$

where $d a$ is area measure on $D$. For $0<p<\infty$ define $T_{\infty}^{p}$ to be the space of functions $u$ continuous on $D$ such that

$$
\|u\|_{T_{\infty}^{p}}=\left(\int_{T}(N u)^{p} d \theta\right)^{\frac{1}{p}}<\infty
$$

where $d \theta$ is arclength measure on $T$ and let $T_{2}^{p}$ be the space of functions $u$ defined on $D$ such that

$$
\|u\|_{T_{2}^{p}}=\left(\int_{T}(A u)^{p} d \theta\right)^{\frac{1}{p}}<\infty .
$$

More generally, for $\alpha>1$ there are maximal functions $N_{\alpha}$ and $A_{\alpha}$ associated with the approach regions

$$
\Gamma_{\alpha}(\zeta)=\left\{z \in D:|1-z \bar{\zeta}|<\frac{\alpha}{2}\left(1-|z|^{2}\right)\right\}
$$

Received by the editors May 28, 1997.

1991 Mathematics Subject Classification. Primary 32A35.

(C)1999 American Mathematical Society 
of aperture $\alpha$. It is shown in [CMS] that the space $T_{\infty}^{p}$ (resp. $T_{2}^{p}$ ) has an equivalent description in terms of $N_{\alpha}$ (resp. $A_{\alpha}$.) We will also need the space $T_{2}^{\infty}$ which is the space of functions $v$ defined on $D$ such that

$$
\|v\|_{T_{2}^{\infty}}=\left(\sup _{I} \frac{1}{|I|} \int_{T(I)}|v(z)|^{2} \frac{d a(z)}{1-|z|}\right)^{\frac{1}{2}}<\infty .
$$

Here, $I$ is an arc on the circle $T, T(I)$ is the "tent" over $I$ (see [CMS]) and $|I|$ is the length of $I$. It is well known that the dual of $T_{2}^{1}$ is $T_{2}^{\infty}$ with the pairing

$$
\langle u, v\rangle=\int_{D} u(z) v(z) \frac{d a(z)}{1-|z|} .
$$

A holomorphic function $f$ defined on $D$ belongs to the Hardy space $H^{p}$ if

$$
\|f\|_{H^{p}}=\left(\int_{T}(N f)^{p} d \theta\right)^{\frac{1}{p}}<\infty .
$$

That is, for $f$ holomorphic in $D$ and $0<p<\infty,\|f\|_{H^{p}}=\|f\|_{T_{\infty}^{p}}$. The norm \|\|$_{H^{\infty}}$ is the usual supremum norm. An alternative characterization of the Hardy spaces $H^{p}$, for $0<p<\infty$ may be given in terms of $T_{2}^{p}$. Suppose that for $\beta>0$ and $f$ holomorphic on $D, R^{\beta}$ is the fractional derivative of $f$ of order $\beta$ :

$$
R^{\beta} f(z)=\sum_{n=0}^{\infty}(1+n)^{\beta} a_{n} z^{n}
$$

where $f(z)=\sum a_{n} z^{n}$ is the Taylor series of $f$. Let $\rho(z)=1-|z|$. It is well known that we have the equivalence of norms

$$
\|f\|_{H^{p}} \doteq|f(0)|+\left\|\rho f^{\prime}\right\|_{T_{2}^{p}},
$$

where the notation $A \doteq B$ means that there is an absolute constant $C$ such that $C^{-1} A \leq B \leq C A$. (We follow the practice of using the letter $C$ to denote various numerical constants whose exact value will depend on the context in which it occurs.) Thus, $f \in H^{p}$ if and only if $\rho R^{1} f \in T_{2}^{p}$. More generally (see [AB]), if $\beta>0$ then a holomorphic function $f$ defined on $D$ belongs to $H^{p}$ for $0<p<\infty$ if and only if $A\left(\rho^{\beta} R^{\beta} f\right) \in L^{p}(d \theta)$. This statement is false if $\beta=0$ : the constant function $f=1$ shows that a function in $H^{p}$ need not belong to $T_{2}^{p}$ (in fact the only such function is the zero function). On the other hand, in [C1] the problem of determining the pointwise multipiers of $H^{p}$ into $T_{2}^{p}$ was investigated. It was shown ([C1], Lemma 1) that if $h \in T_{2}^{\infty}$ then $h$ has the property that $h f \in T_{2}^{p}$ for all functions $f \in H^{p}$. In fact the argument used in that proof establishes the following inequality.

Theorem A. Let $0<p<\infty$. There is a constant $C$ depending only on $p$ such that

$$
\|u h\|_{T_{2}^{p}} \leq C\|u\|_{T_{\infty}^{p} \|}\|h\|_{T_{2}^{\infty}},
$$

for all $u \in T_{2}^{p}$ and $h \in T_{2}^{\infty}$.

A type of converse to this result was given in [C2]. See also [CMS], pg. 320, and Remark 1 below.

Recall that one of the many characterizations of the class $B M O A$ of holomorphic functions on $D$ which have bounded mean oscillation is in terms of Carleson 
measures and the space $T_{2}^{\infty}$. A holomorphic function $\Phi \in B M O A$ if and only if the function $\rho \Phi^{\prime} \in T_{2}^{\infty}$. Furthermore,

$$
\|\Phi\|_{B M O A}=|\Phi(0)|+\left\|\rho \Phi^{\prime}\right\|_{T_{2}^{\infty}}
$$

defines a norm equivalent to the intrinsic norm which is given in terms of $\Phi(0)$ and mean oscillation on arcs. See $[G]$. We therefore have the following fact.

Let $\Phi \in B M O A$. Then there is a constant $C(p)$ such that

$$
\left\|\rho f \Phi^{\prime}\right\|_{T_{2}^{p}} \leq C(p)\|f\|_{T_{\infty}^{p}}\|\Phi\|_{B M O A}
$$

The characterization of $H^{p}$ in terms of $T_{2}^{p}$ given by (2) combined with (3) yields the following corollary.

Corollary B. Let $\Phi \in B M O A, F \in H^{p}$, and $0<p<\infty$. Then $F \Phi^{\prime}$ is the derivative of an $H^{p}$ function $f$ with $\|f\|_{H^{p}} \leq C\|F\|_{H^{p}}\|\Phi\|_{B M O A}$.

In this note we prove the converse of this statement.

Theorem 1. Let $0<p<\infty$. A function $G$ holomorphic in $D$ is of the form $G=f^{\prime}$ for $f \in H^{p}$ if and only if $G=F \Phi^{\prime}$ where $F \in H^{p}$ and $\Phi \in B M O A$. Furthermore, $F$ and $\Phi$ may be chosen so $F$ is non-vanishing and outer in $D, \Phi(0)=0,\|\Phi\|_{B M O A} \leq$ 1 and $\|F\|_{H^{p}} \leq C(p)\|f\|_{H^{p}}$.

Remark 1. In [CMS], pg. 320, it was observed that for $p>2$, a function $u \in T_{2}^{p}$ if and only if $u=u_{1} u_{2}$ where $u \in T_{\infty}^{p}$ and $u_{2} \in T_{2}^{\infty}$. Thus Theorem 1 may regarded as a holomorphic version of this factorization.

Remark 2. For $0<p, q<\infty$ let $F^{p, q}$ be the space of holomorphic functions $G$ defined on $D$ such that

$$
\|G\|_{F^{p, q}}=\left(\int_{0}^{2 \pi}\left(\int_{0}^{1}\left|G\left(r e^{i \theta}\right)\right|^{q}(1-r)^{q-1} d r\right)^{\frac{p}{q}} d \theta\right)^{\frac{1}{p}}<\infty .
$$

If $q=2$, then $G \in F^{p, q}$ if and only if $G=f^{\prime}$ for some $f \in H^{p}$. Since given $f \in H^{p}$ we may write $f^{\prime}=F \Phi^{\prime}$ with $F$ non-vanishing, it follows easily that the zero sets of functions in the classes $F^{p, 2}, 0<p<\infty$, coincide with the zero sets of derivatives of functions in the class $B M O A$. This result is in contrast to the result of Horowitz $[\mathrm{H}]$, Theorem 1, that given $p<q$ there is a zero set for the Bergman space $A^{p}$ which is not a zero set for the Bergman space $A^{q}$. (See $[\mathrm{H}]$ for the relevant definitions.) It would be interesting to find a similar factorization theorem and results about zeros sets for the spaces $F^{p, q}, q \neq 2$.

As a second application of Theorem 1 we study the operator

$$
L_{\mu} f(\zeta)=\int_{D} \frac{f(z)}{(1-\zeta \bar{z})^{2}} d \mu(z)
$$

where $\mu$ is a positive Borel measure defined on $D$. With the aid of Theorem 1 (and an appeal to a result of Luecking [Lu], Theorem 1) we characterize all measures $\mu$ with the property that $L_{\mu}$ defines a bounded operator from $T_{\infty}^{p}$ into $H^{p}$ if $\frac{1}{2}<p<\infty$. For $z \in D$ and $0<t<1$ let $D_{t}(z)=\{w \in D:|z-w|<t(1-|z|)\}$. For a measure $\mu$ defined on $D$ let $H^{t} \mu$ be the function defined by

$$
H^{t} \mu(z)=\frac{1}{(1-|z|)^{2}} \int_{D_{t}(z)} d \mu
$$


Theorem 2. Let $\frac{1}{2}<p<\infty$ and $\mu$ a positive Borel measure defined on D. Then the operator $L_{\mu}$ is bounded from $T_{\infty}^{p}$ into $H^{p}$ if and only if $H^{t} \mu \in T_{2}^{\infty}$ for some $0<t<1$.

The proof of Theorem 1 is based on the following simple observation.

Lemma 3. Suppose $F$ is a holomorphic on $D$ with positive real part. Then there is a constant $C$ independent of $F$ such that $\left\|\rho \frac{F^{\prime}}{F}\right\|_{T_{2}^{\infty}} \leq C$. Furthermore, let $\log F$ be a logarithm of $F$ with the property that $|\operatorname{Im} \log F(0)|<\frac{\pi}{2}$. Then $\log F \in B M O A$ and there is a constant $C$ independent of $F$ such that $\|\log F\|_{B M O A} \leq C$.

Proof. Since $\operatorname{Re} F(z)>0,|\operatorname{Im} \log F(z)|<\frac{\pi}{2}$ for all $z \in D$. The result follows since the Hilbert transform is a bounded operator from $L^{\infty}$ into $B M O$. See $[\mathrm{G}]$, chapter VI, Theorem 1.5.

Proof of Theorem 1. We now give the proof of Theorem 1. We need only show that if $G=f^{\prime}$ where $f \in H^{p}$ then $G=F \Phi^{\prime}$ where $F$ and $\Phi$ are as in the statement of the theorem. We consider first the case where $p>1$. Let $f \in H^{p}$. Then $|f| \in L^{p}(d \theta)$. Since $1<p<\infty$, by the Marcel Riesz theorem (see [G], chapter 2, Theorem 2.3) we may find a holomorphic function $F$ whose real part is the Poisson integral of $|f|$ such that $\|F\|_{H^{p}} \leq C\|f\|_{H^{p}}$. Here, $C$ depends only on $p$. Note that $\operatorname{Re} F(z) \geq|f(z)|$ for all $z \in D$. Write

$$
f=F \frac{f}{F}=F \psi,
$$

where $\psi=\frac{f}{F}$. Then $\psi \in H^{\infty},\|\psi\|_{\infty} \leq 1$, and

$$
\begin{aligned}
f^{\prime} & =F^{\prime} \psi+F \psi^{\prime} \\
& =F\left(\psi^{\prime}+\psi \frac{F^{\prime}}{F}\right) \\
& =F\left(\psi^{\prime}+\psi(\log F)^{\prime}\right) .
\end{aligned}
$$

Let $\Phi$ be defined by the two conditions $\Phi^{\prime}=\psi^{\prime}+\psi(\log F)^{\prime}$ and $\Phi(0)=0$. Then the conclusion of the theorem (for the case $1<p<\infty$ ) follows from Lemma 3 .

We next consider the case where $0<p<2$. Let $G=f^{\prime}$ where $f \in H^{p}$ and factor $f$ as $f=B H^{\frac{2}{p}}$ where $B$ is an inner function and $H$ is an outer function with $H \in H^{2}$ with $\|H\|_{H^{2}}^{2}=\|f\|_{H^{p}}^{p}$. As before, we may find $F$ so $\operatorname{Re} F \geq|H|$ and $\|F\|_{H^{2}} \leq C\|H\|_{H^{2}}$, where $C$ depends only on $p$. Therefore

$$
\begin{aligned}
f & =B\left(\frac{H}{F}\right)^{\frac{2}{p}} F^{\frac{2}{p}} \\
& =\psi F^{\frac{2}{p}},
\end{aligned}
$$

where $\|\psi\|_{\infty} \leq 1$ and $\left\|F^{\frac{2}{p}}\right\|_{H^{p}} \leq C\|f\|_{H^{p}}$. Thus

$$
\begin{aligned}
f^{\prime} & =\psi^{\prime} F^{\frac{2}{p}}+\frac{2}{p} F^{\frac{2}{p}-1} F^{\prime} \psi \\
& =F^{\frac{2}{p}}\left(\psi^{\prime}+\frac{2}{p} \frac{F^{\prime}}{F} \psi\right) \\
& =F^{\frac{2}{p}}\left(\psi^{\prime}+\frac{2}{p}(\log F)^{\prime} \psi\right)
\end{aligned}
$$

and the proof may be completed as in the first case. 
Proof of Theorem 2. We now give the proof of Theorem 2. For $z \in D$ and $0<t<1$ recall that $D_{t}(z)=\{w \in D:|z-w|<t(1-|z|)\}$ and if $\mu$ is a positive measure defined on $D$, then

$$
H^{t} \mu(z)=\frac{1}{(1-|z|)^{2}} \int_{D_{t}(z)} d \mu .
$$

For a function $u$ defined on $D$ let $M^{t} u(z)=\sup _{w \in D_{t}(z)}|u(w)|$.

Suppose that $H^{t} \mu \in T_{2}^{\infty}$ for some $t>0$. We show that if $1 / 2<p<\infty$, then $L_{\mu}$ is a bounded operator from $T_{\infty}^{p}$ to $H^{p}$.

Consider first the case where $p>1$. By duality, it suffices to show there is a constant $C$ independent of $g$ or $f$ such that

$$
\left|\int_{T} \overline{\left(L_{\mu} g\right)(\zeta)} f(\zeta) d \zeta\right| \leq C|| g\left\|_{T_{\infty}^{p}}\right\| f \|_{H^{p^{\prime}}}
$$

where $p^{\prime}$ is the exponent conjugate to $p$. Interchanging the order of integration in the integral in (5) shows that it is enough to prove that

$$
\int_{D}\left|g(z) f^{\prime}(z)\right| d \mu(z) \leq C\|g\|_{T_{\infty}^{p}}\|f\|_{H^{p \prime}}
$$

Write $f^{\prime}=F \Phi^{\prime}$ where $F$ and $\Phi$ are as in Theorem 1. Since the spaces $T_{\infty}^{p}$ are independent of the aperture of the approach region, it is easily verified that for $t<1$ there is a constant depending on $t$ but not $g$ or $f$ such that

$$
\left\|M^{t}(g F)\right\|_{T_{\infty}^{1}} \leq C\|g\|_{T_{\infty}^{p}}\|f\|_{H^{p^{\prime}}} .
$$

Furthermore, since $\left|\Phi^{\prime}\right|^{2}$ is subharmonic, there is also a constant $C$ depending only on $t$ such that

$$
\left\|\rho M^{t}\left(\Phi^{\prime}\right)\right\|_{T_{2}^{\infty}} \leq C .
$$

Let $G(z)=\left|g(z) f^{\prime}(z)\right|=\left|g(z) F(z) \Phi^{\prime}(z)\right|$. It follows from Theorem A that for any $t<1$, there is a constant $C$ depending only on $t$ and $p$ such that

$$
\left\|\rho M^{t} G\right\|_{T_{2}^{1}} \leq\left. C\|g\|\right|_{T_{\infty}^{p}}\|f\|_{H^{p}} .
$$

For a function $u$ defined on $D$ and $0<t<1$ let $H^{t} u=H^{t}(u d a)$. It is easily seen that we may choose $s$, independent of $G, 0<s<t$, so

$$
G(z) \leq C H^{s}\left(M^{t} G\right)(z)
$$

for all $z \in D$. It follows from Fubini's theorem and the duality given by the pairing (1) that

$$
\begin{aligned}
\int_{D}\left|g(z) f^{\prime}(z)\right| d \mu(z) & =\int_{D} G(z) d \mu(z) \\
& \leq C \int_{D} H^{s}\left(M^{t} G\right)(z) d \mu(z) \\
& \leq C \int_{D} M^{t} G(z) H^{s} \mu(z) d a(z) \\
& =C \int_{D} \rho(z) M^{t} G(z) H^{s} \mu(z) \frac{d a(z)}{1-|z|} \\
& \leq C|| \rho M^{t} G\left\|_{T_{2}^{1}}|| H^{s} \mu\right\|_{T_{2}^{\infty}},
\end{aligned}
$$

which implies the desired inequality since $H^{s} \mu \leq H^{t} \mu$. 
We consider next the case where $1 / 2<p \leq 1$. Using the atomic decomposition for $T_{\infty}^{p}$ (see [CMS]) it suffices to prove that there is a constant $C$ such that for any arc $I$ contained in $T$ with $|I| \leq \frac{1}{4}$ and any function $b$ supported in $T(I)$ and bounded by 1 the inequality

$$
\int_{T}\left|L_{\mu} b\right|^{p} d \theta \leq C|I|
$$

is verified. Write the integral on the left as a sum

$$
\int_{T}=\int_{3 I}+\int_{T-3 I}
$$

where $C I$ is an interval with the same center as $I$ and $C$ times the radius. Then by the result proved above in the case $p=2$

$$
\begin{aligned}
\left|\int_{3 I}\right| & \leq C\left(\int_{3 I}\left|L_{\mu} b\right|^{2} d \theta\right)^{\frac{p}{2}}(|I|)^{1-\frac{p}{2}} \\
& \leq C\left(\left.|| b\right|_{T_{2}^{2}} ^{2}\right)^{\frac{2}{p}}(|I|)^{1-\frac{p}{2}} \\
& \leq C|I|^{\frac{2}{p}}|I|^{1-\frac{p}{2}}=C|I|
\end{aligned}
$$

which is the needed estimate.

For the second integral, let $\eta$ be the center of $I$ and estimate from (4) that for $\zeta \in T-3 I$

$$
\left|L_{\mu} b(\zeta)\right| \leq|1-\zeta \bar{\eta}|^{-2} \int_{T(I)} d \mu .
$$

Observe that for all $z \in D, 1 \leq C H^{t} 1(z)$ for a constant $C$ depending only on $t$. Furthermore, if $t<1$, then there is a constant $C$, again depending only on $t$ such that the set $\{w \in D:|z-w| \leq t(1-|z|)$ and $z \in T(I)\}$ is contained in $T(C I)$. These two facts together with Fubini's theorem show that

$$
\begin{aligned}
\int_{T(I)} d \mu & \leq C \int_{T(I)} H^{t} 1 d \mu \\
& \leq C \int_{T(C I)} H^{t} \mu(z) d a(z) \\
& \leq C\left(\int_{T(C I)}(1-|z|) d a(z)\right)^{\frac{1}{2}}\left(\int_{T(C I)}\left(H^{t} \mu(z)\right)^{2} \frac{d a(z)}{1-|z|^{\frac{1}{2}}}\right. \\
& \leq C|I|^{\frac{3}{2}}|I|^{\frac{1}{2}}=|I|^{2},
\end{aligned}
$$

where we have used Holder's inequality to obtain the third estimate and the fact that $H_{\mu}^{t} \in T_{2}^{\infty}$ to obtain the fourth estimate. Thus, for $\zeta \in T-3 I$,

$$
\left|L_{\mu} b(\zeta)\right| \leq C|I|^{2}|1-\zeta \bar{\eta}|^{-2}
$$

Taking $p^{\text {th }}$ powers and integrating over $T-3 I$ gives the needed estimate.

Suppose now that $\mu$ is a positive measure and $L_{\mu}$ defines a bounded operator from $T_{\infty}^{p}$ into $H^{p}$. Consider first the case where $1<p<\infty$. By duality we may start with (5) and interchange the order of integration as before to see that there is a constant $C=C(p)$ such that

$$
\left|\int_{D} g(z) f^{\prime}(z) d \mu(z)\right| \leq C|| g\left\|_{T_{\infty}^{p}}\right\| f \|_{H^{p^{\prime}}}
$$


and it follows from this and Corollary B that for all $g \in T_{\infty}^{p}, F \in H^{p}$ and all $\psi \in B M O A$

$$
\int_{D}\left|g(z) F(z) \psi^{\prime}(z)\right| d \mu(z) \leq C\|g\|_{T_{\infty}^{p}}\|F\|_{H^{p^{\prime}}}\|\psi\|_{B M O A} .
$$

Therefore Theorem 1 yields the inequality

$$
\int_{D}\left|H^{\prime}(z)\right| d \mu(z) \leq C|| H \|_{H^{1}}
$$

for all $H \in H^{1}$ and the result follows from Theorem 1 of $[\mathrm{Lu}]$.

Consider now the case where $\frac{1}{2}<p \leq 1$. Let $I$ be an arc on $T$ with $|I| \leq \frac{1}{4}$. If $L_{\mu}$ is a bounded operator from $T_{\infty}^{p}$ into $H^{p}$ then there is a constant $C=C(p)$ independent of $I$ such that $\left\|T_{\mu} b\right\|_{H^{p}}^{p} \leq C|I|$ for any continuous function $b$ supported in $T(3 I)$ bounded by 1 . From this it follows that there is a constant $C=C(p)$ independent of $I$ such that

$$
\frac{1}{|I|} \int_{I}\left|\int_{T(3 I)} \frac{d \mu(z)}{\left(1-e^{i \theta} \bar{z}\right)^{2}}\right|^{p} d \theta \leq C
$$

By bounding the real part of the inside integral in (6) from below it can be shown that there is a number $t, 0<t<1$, and a constant $C=C(t)$ such that

$$
\mu\left(D_{t}(z)\right) \leq C(1-|z|)^{2}
$$

for all $z \in D$. Let

$$
F(\zeta)=L_{\mu} 1(\zeta)=\int_{D} \frac{d \mu(z)}{(1-\zeta \bar{z})^{2}}
$$

Claim. The function $F$ belongs to BMOA.

To see this, define $F_{I}$ and $G_{I}$ by

$$
F_{I}(\zeta)=\int_{T(3 I)} \frac{d \mu(z)}{(1-\zeta \bar{z})^{2}}
$$

and $G_{I}=F-F_{I}$. The following lemma depends essentially on the estimate (7).

Lemma 4. There is a constant $C$ such that for all arcs $I$ contained in $T$

$$
\left|G_{I}(\zeta)-G_{I}\left(\zeta^{\prime}\right)\right| \leq C
$$

for all $\zeta, \zeta^{\prime}$ in $I$.

Proof. Let $\eta$ be the center of $I$. There is a constant $C$ independent of $I$ such that with $\zeta$ and $\zeta^{\prime}$ as in the lemma and $z \in D-T(3 I)$

$$
\left|\frac{1}{(1-\zeta \bar{z})^{2}}-\frac{1}{\left(1-\zeta^{\prime} \bar{z}\right)^{2}}\right| \leq C \frac{|I|}{|1-\eta \bar{z}|^{3}} .
$$


It follows that

$$
\begin{aligned}
\left|G_{I}(\zeta)-G_{I}\left(\zeta^{\prime}\right)\right| & =\left|\int_{D-T(3 I)} \frac{1}{(1-\zeta \bar{z})^{2}}-\frac{1}{\left(1-\zeta^{\prime} \bar{z}\right)^{2}} d \mu(z)\right| \\
& \leq C \int_{D-T(3 I)} \frac{|I|}{|1-\eta \bar{z}|^{3}} d \mu(z) \\
& \leq C \int_{D-T(3 I)} \frac{|I|}{|1-\eta \bar{z}|^{3}} d a(z) \\
& \leq C
\end{aligned}
$$

where we used (7) to replace $d \mu$ with $d a$ in the third line. This proves the lemma.

It follows from Lemma 4 that for any arc $I$ in $T$ there is a constant $C$, independent of $I$, a function $b_{I}$ and a constant $c_{I}$ both bounded by $C$ such that

$$
G_{I} \chi_{I}=\left(c_{I}+b_{I}\right) \chi_{I},
$$

where $\chi_{I}$ is the characteristic function of $I$.

We can now prove the claim. Since $F=G_{I}+F_{I}$ it follows that

$$
\begin{aligned}
F \chi_{I} & =\left(G_{I}+F_{I}\right) \chi_{I} \\
& =\left(c_{I}+b_{I}\right) \chi_{I}+F_{I} \chi_{I},
\end{aligned}
$$

and therefore $\left(F-c_{I}\right) \chi_{I}=b_{I} \chi_{I}+F_{I} \chi_{I}$. It follows from this and (6) that

$$
\int_{I}\left|F-c_{I}\right|^{p} d \theta \leq C|I|
$$

and this proves the claim.

The same argument shows that if $b$ is any bounded function, then there is a constant $C$ such that

$$
\left\|L_{\mu} b\right\|_{B M O A} \leq(C+\mu(D))\|b\|_{\infty} .
$$

Therefore, if $F \in H^{1}$ it follows that there is a constant $C$ such that

$$
\left|\int_{T} F \overline{L_{\mu} b} d \theta\right| \leq C|| F\left\|_{H^{1}}\right\| b \|_{\infty}
$$

for all $F \in H^{1}$. Interchanging the order of integration in the integration on the left hand side yields the inequality

$$
\int_{D}\left|F^{\prime}(z)\right| d \mu(z) \leq C\|F\|_{H^{1}}
$$

for all $F \in H^{1}$. The desired result follows now by Theorem 1 of [Lu].

\section{REFERENCES}

[AB] P. Ahern and J. Bruna, Maximal and area integral characterizations of Hardy-Sobolev spaces in the unit ball of $\mathbf{C}^{n}$, Revista Mat. IberoAmer., vol. 4, no.1 (1988), 123-153. MR 90h:32011

[CMS] R. Coifman, Y. Meyer, E. Stein, Some new function spaces and their applications to harmonic analysis, J. Funct. Anal., 62 (1985), 304-335. MR 86i:46029

[C1] W. Cohn, Weighted Bergman projections and operators on Hardy spaces, J. Funct. Anal. 144, No.1, (1997), 1-19. MR 98a:47033

[C2] W. Cohn, Generalized area operators on Hardy spaces, J. Math. Anal. Appl., 216 (1997), $112-121$.

[G] J. Garnett, "Bounded Analytic Functions", Academic Press, New York/London, 1982. MR 83g:30037 
[H] C. Horowitz, Zeros of functions in the Bergman space, Duke Math. J., 41, (1974), 693-710. MR 50:10215

[Lu] D. H. Luecking, Embedding derivatives of Hardy spaces into Lebesgue spaces, Proc. London Math. Soc., 63, no.3 (1991), 595-619. MR 92k:42030

Department of Mathematics, Wayne State University, Detroit, Michigan 48070 